\title{
Effects of various interventions on the occurrence of macrovascular invasion of hepatocellular carcinoma after the baseline serum $\gamma$-glutamyltransferase stratification
}

This article was published in the following Dove Medical Press journal: OncoTargets and Therapy

Yao Liu'
Qun Zhang'
Xue Yang'
Yuxin Li'
Bingbing Zhu'
Shuaishuai Niu'
Yunyi Huang'
Ying Hu'
Xianbo Wang'
'Center of Integrative Medicine,
Beijing Ditan Hospital, Capital Medical
University, Beijing I000I5, People's
Republic of China; ${ }^{2}$ Department
of Gastroenterology, Dongzhimen
Hospital, Beijing University of Chinese
Medicine, Beijing I00700, People's
Republic of China

Correspondence: Xianbo Wang Center of Integrative Medicine, Beijing Ditan Hospital, Capital Medical University, No 8, Jing Shun East Street, Beijing I00015, People's Republic of China

Tel/fax +86 I0 84322301

Email wangxb@ccmu.edu.cn

\begin{abstract}
Background: Elevated serum $\gamma$-glutamyltransferase $(\gamma$-GT) levels are related to an increased cancer risk and worse prognosis in many cancers. We evaluated the effects of $\gamma$-GT stratification on the occurrence of macrovascular invasion (MVI) in patients with hepatocellular carcinoma (HCC) who underwent hepatic resection (HR), transcatheter arterial chemoembolization (TACE), or TACE combined with radiofrequency ablation (TACE-RFA).

Patients and methods: A total of 903 patients with HCC in Barcelona Clinic Liver Cancer Stage A or B were included. Of these patients, 118 underwent HR, 445 underwent TACE-RFA, 256 underwent TACE, and 84 patients received conservative treatment only (control group). $\gamma$-GT, albumin, $\alpha$-fetoprotein, and intervention were selected as significant predictive factors for MVI in 1 year by forward selection. The optimal cutoff value of $\gamma$-GT was 39 IU/L according to receiver operating characteristic analysis, with a sensitivity and specificity of $87.0 \%$ and $45.6 \%$, respectively.

Results: The 1-year MVI incidence of patients with HCC in the group with $\gamma$-GT $\geq 39$ IU/L was higher than that of the group with $\gamma$-GT $<39$ IU/L treated with HR, TACE-RFA, or TACE ( $P=0.0166, P=0.0041$, and $P<0.001$, respectively). The MVI rates at 1 year were similar in the group with $\gamma$-GT $\geq 39 \mathrm{IU} / \mathrm{L}$ that underwent HR, TACE-RFA, or TACE and the control group ( $P=0.4402, P=0.2214$, and $P=0.4159$, respectively). Different effects of various treatments with $\gamma$-GT $<39$ IU/L group on the occurrence of MVI are not significant $(P=0.5167)$. However, the incidence of MVI after TACE was significantly higher than that after HR or TACE-RFA in $\gamma$-GT $\geq 39$ IU/L group $(P=0.0253)$.
\end{abstract}

Conclusion: Baseline serum $\gamma$-GT stratification may help select the appropriate treatment to reduce the MVI incidence.

Keywords: gamma-glutamyltransferase, macroscopic vascular invasion, liver cancer

\section{Introduction}

One of the most common malignancies, hepatocellular carcinoma (HCC) is ranked the second most frequent cause of deaths for cancer and the fifth most common cancer based on global cancer statistics. ${ }^{1}$ Patients with HCC can achieve a 5-year survival rate of $\sim 70 \%$ with liver transplantation, radiofrequency ablation (RFA), or curative hepatic resection (HR) in the early tumor stage., ${ }^{2,3}$ Long-term survival remains far from satisfactory owing to a high rate of cancer recurrence. ${ }^{4}$

Macrovascular invasion (MVI), which includes the presence of a tumor thrombus in the portal, hepatic, and/or inferior vena cava vein, is the most important negative 
risk factor for survival after resection in patients with HCC. ${ }^{5}$ Furthermore, in patients with non-resectable HCC, MVI significantly reduced the median survival time (2-4 months) compared to the survival time in patients without MVI (10-24 months).$^{6-8}$ Some clinical pathological factors, including tumor size and poor differentiation and some molecular biomarkers such as $\alpha$-fetoprotein (AFP) and glypican-3 have been confirmed as prognostic predictors of HCC. ${ }^{9-12}$ However, confirmation of other predictive factors to identify the patients with HCC who have a high risk of MVI is important to improve the prognosis of these patients using novel treatments and clinical decision-making surveillance plans.

Currently, MVI can only be diagnosed through medical imaging examination such as ultrasound, computed tomography (CT), magnetic resonance imaging (MRI), and angiography. Previous studies have shown that tumor size, Edmondson-Steiner histological grade, number of nodules, and AFP level are associated with portal vein tumor thrombosis (PVTT), ${ }^{13}$ whereas low albumin (ALB) levels, tumor size of $>5 \mathrm{~cm}$, metastases, ascites, AFP $>1,000 \mathrm{ng} / \mathrm{mL}$, and hypersplenism have been found to be independent predictive factors of microvascular invasion, ${ }^{14-16}$ however, these indicators cannot predict the risk of MVI after various interventions.

As a membrane-bound enzyme, $\gamma$-glutamyltransferase $(\gamma-\mathrm{GT})$ is involved in glutathione metabolism. ${ }^{17}$ Serum $\gamma$-GT is a marker of hepatic injury, and its levels are elevated by alcohol consumption, acute and chronic liver disease, and oxidative stress. ${ }^{18-20}$ Moreover, it has been reported that $\gamma$-GT has a crucial function in carcinogenesis via prooxidant effects at the membrane surface level and in the extracellular microenvironment and by increasing free iron release from transferrin. ${ }^{21} \gamma$-GT is one of the important risk factors for cancer, tumor progression, invasion, and anticancer drug resistance. $^{22-24}$ Regarding HCC, elevated serum $\gamma$-GT has been reported as a biomarker of poor prognosis after hepatectomy, transcatheter arterial chemoembolization (TACE), and RFA. ${ }^{25-28}$ However, there has been no study on the predictive roles of $\gamma$-GT for MVI in patients with HCC without MVI undergoing different interventions. It can be assumed that $\gamma$-GT may represent a predictive factor for MVI. This hypothesis was tested by investigating the association of baseline serum $\gamma$-GT with the incidence of MVI in 903 patients with HCC who underwent HR, TACE combined with RFA (TACE-RFA), TACE, or conservative treatment only.

\section{Patients and methods}

\section{Patients and treatments}

The patients with HCC, whose clinical data were collected, were initially treated at the Beijing Ditan Hospital (Beijing,
China), Capital Medical University from October 2008 to May 2016. HCC was diagnosed based on recommendations of the American Association for Liver Diseases Study. ${ }^{29}$ A routine workup was performed before interventions, including a comprehensive physical examination, hematologic and biochemistry profiles, chest radiography or CT, and an electrocardiograph. No patient had any treatment before the interventions.

A total of 903 patients underwent HR $(n=118)$, TACERFA ( $n=445)$, TACE $(n=256)$, or conservative treatment only (control, $n=84$ ). The control group received no systemic chemotherapy, locoregional therapies, or surgical treatment. Instead, they received symptomatic supportive care mainly to treat complications, such as ascites, hypersplenism, upper gastrointestinal bleeding, hepatorenal syndrome, and hepatic failure. The patient selection criteria for inclusion in our research were as follows: 1) Barcelona Clinic Liver Cancer (BCLC) A or B stage with no extrahepatic metastasis and 2) hepatitis B virus, hepatitis $C$ virus, and/or alcohol related to the etiology of HCC. Patients were excluded if they had autoimmune liver disease, hepatitis A, D, or E, syphilis, AIDS, or if they had incomplete data or lacked follow-up. Our multidisciplinary team selected the treatments. The default treatment was HR or TACE-RFA; patients underwent TACE upon request or if they had diffused HCC based on gross pathological findings.

The Ethics Committee of the Beijing Ditan Hospital, Capital Medical University (Beijing, China) approved this study. Because this study has a retrospective design, we could not obtain informed consent from all patients. However, to protect patient privacy, we anonymized and deidentified all patient records and information before the analysis.

\section{Follow-up}

To evaluate the efficacy of the techniques, patients underwent contrast-enhanced CT (CECT) or enhanced MRI at 4 weeks after treatment. Thus, over the course of 1 year, patients were treated once every 3 months. At each follow-up visit, patients underwent CECT or enhanced MRI, chest CT, and blood tests including liver function tests and AFP test. The MVI was diagnosed using CECT or enhanced MRI when a filling defect was shown in the portal vein, hepatic vein, or inferior vena cava, and the emboli were enhanced in the similar way as the primary liver cancer. ${ }^{30}$

Patients with local lesion recurrence or intrahepatic distant or extrahepatic recurrence during follow-up received corresponding treatments such as resection, RFA, TACE, sorafenib, and conservative treatments based on the recurrent 
tumor characteristics, liver function status, and patient request. The duration of MVI was the interval between the time MVI occurred after the first treatment and the time of death or the last follow-up.

\section{Statistical analysis}

Continuous variables are presented as mean $\pm \mathrm{SD}$, discontinuous variables as median and range, and categorical variables as numbers and percentages. $\gamma$-GT data have been converted into categorical variables based on the cutoff values, calculated based on the maximum Youden index (sensitivity + specificity -1 ) values. The baseline covariates were compared to the paired $t$-test or the MannWhitney $U$ test for continuous variables and chi-squared test for categorical variables. Univariate and multivariate Cox proportional hazards regression analyses were used to identify significant factors predicting the risk of MVI. The receiver operating characteristics (ROC) curve was used to compare the predictive accuracy of the various factors. A cutoff value for the maximum sensitivity and specificity of $\gamma$-GT was calculated and used to divide the patients into two groups based on that value. MVI incidence curves were constructed using the Kaplan-Meier method and compared using the log-rank test. SPSS version 22.0 statistical package was used for statistical analyses (IBM Corporation, Armonk, NY, USA). All $P$-values $<0.05$ were considered significant.

\section{Results \\ Characteristics of patients according to intervention}

The baseline characteristics of 903 patients with HCC are shown in Table 1. The age of patients who underwent HR ranged from 29 to 74 years, with a median age of 52 years, which was lower than that of the other groups. The majority of patients were male in all groups. Patients who received HR and TACE-RFA had lower $\gamma$-GT and total bilirubin (TBIL) levels than the conservative treatment group. The ALB level was lower in the control group than in the three intervention groups. Child-Pugh class A accounted for $58.3 \%$ of the control group, $72.0 \%$ of the HR group, $76.6 \%$ of the TACERFA group, and $66.8 \%$ of the TACE group. The Models for End-Stage Liver Disease Score (MELD) score was lowest in the HR group. The four different intervention groups had a similar tumor number and tumor diameter.

The recurrence rates for HR, TACE-RFA, and TACE cohorts at 12 months were $16.9 \%$ (20 of 118 ), $27.2 \%$ (121 of 445 ), and $42.2 \%$ (108 of 256), respectively. The MVI rates for HR, TACE-RFA, and TACE cohorts at 12 months were $4.2 \%$ (5 of 118), 5.8\% (26 of 445), and 10.5\% (27 of 256), respectively.

\section{Predictive factors for MVI}

We evaluated the prognostic value of 13 variables to identify predictors of MVI. Univariate Cox regression analyses

Table I Patients' baseline characteristics

\begin{tabular}{|c|c|c|c|c|c|c|c|}
\hline \multirow[t]{2}{*}{ Variables } & \multirow{2}{*}{$\begin{array}{l}\text { Control } \\
n=84\end{array}$} & \multicolumn{2}{|l|}{ HR } & \multicolumn{2}{|l|}{ TACE-RFA } & \multicolumn{2}{|l|}{ TACE } \\
\hline & & $n=118$ & $P$-value & $n=445$ & $P$-value & $\mathrm{n}=\mathbf{2 5 6}$ & $P$-value \\
\hline Median age, years (range) & $58(36-78)$ & $52(29-74)$ & $<0.001^{\mathrm{a}}$ & $57(28-8 I)$ & $0.372^{\mathrm{a}}$ & $57(33-84)$ & $0.634^{\mathrm{a}}$ \\
\hline $\operatorname{Sex}(M / F)$ & $69 / 15$ & $97 / 21$ & $0.991^{b}$ & $346 / 99$ & $0.369^{b}$ & $|95 / 6|$ & $0.254^{b}$ \\
\hline HBV related (yes/no) & $73 / 11$ & $106 / 12$ & $0.367^{b}$ & $381 / 64$ & $0.756^{\mathrm{b}}$ & $214 / 42$ & $0.468^{b}$ \\
\hline ALT (IU/L) & $36.1(24.4-52.8)$ & $32.0(23.4-49.8)$ & $0.295^{c}$ & $31.0(21.4-49.8)$ & $0.128^{c}$ & $32.1(21.1-48.9)$ & $0.176^{c}$ \\
\hline AST (IU/L) & $44.6(28.6-68.8)$ & $35.9(26.6-56.1)$ & $0.025^{c}$ & $34.5(25.0-55.2)$ & $0.00 I^{c}$ & $36.8(25.9-55.6)$ & $0.017^{c}$ \\
\hline ALP (IU/L) & $90.8(77.0-117.4)$ & $85.8(68.4-118.3)$ & $0.112^{c}$ & $84.7(65.2-112)$ & $0.024^{c}$ & $85.5(65.1-117.2)$ & $0.090^{c}$ \\
\hline$\gamma-\mathrm{GT}(\mathrm{IU} / \mathrm{L})$ & $52.1(26.4-92.4)$ & $36.8(22.3-80.1)$ & $0.040^{c}$ & $46.6(26.7-89.0)$ & $0.002^{c}$ & $45.2(26.4-86.1)$ & $0.329^{c}$ \\
\hline TBIL $(\mu \mathrm{mol} / \mathrm{L})$ & $19.5(\mid 4.0-35.0)$ & $15.7(11.3-23.5)$ & $0.012^{c}$ & $16.2(11.3-23.2)$ & $0.002^{c}$ & $17.9(12.5-27.7)$ & $0.14 I^{c}$ \\
\hline ALB $(g / L)($ mean $\pm S D)$ & $34.6 \pm 7.2$ & $37.3 \pm 7.2$ & $0.008^{a}$ & $37.4 \pm 6.8$ & $<0.00 \mathrm{I}^{\mathrm{a}}$ & $37.0 \pm 6.5$ & $0.004^{\mathrm{a}}$ \\
\hline Child-Pugh class (A/B) & $49 / 35$ & $85 / 33$ & $0.042^{b}$ & $34 I / 104$ & $<0.00 \mathrm{I}^{\mathrm{b}}$ & $17 \mid / 85$ & $0.159^{b}$ \\
\hline $\mathrm{PT}($ mean $\pm \mathrm{SD})$ & $13.9 \pm 2.6$ & $13.3 \pm 2.3$ & $0.108^{\mathrm{a}}$ & $13.0 \pm 1.9$ & $0.003^{\mathrm{a}}$ & $13.3 \pm 2.2$ & $0.057^{\mathrm{a}}$ \\
\hline MELD score & $6.6 \pm 5.7$ & $4.4 \pm 3.5$ & $0.002^{\mathrm{a}}$ & $5.4 \pm 4.1$ & $0.077^{a}$ & $6.0 \pm 4.7$ & $0.369^{c}$ \\
\hline $\operatorname{AFP}(\mathrm{ng} / \mathrm{mL})(<400 / \geq 400)$ & $64 / 20$ & $101 / 17$ & $0.089^{b}$ & $383 / 62$ & $0.022^{b}$ & $210 / 46$ & $0.240^{\mathrm{b}}$ \\
\hline Tumor number $(<3 / \geq 3)$ & $63 / 21$ & $92 / 26$ & $0.623^{b}$ & $347 / 98$ & $0.549^{b}$ & $183 / 73$ & $0.532^{\mathrm{b}}$ \\
\hline Tumor diameter $(<5 / \geq 5 \mathrm{~cm})$ & $69 / 15$ & $99 / 19$ & $0.742^{b}$ & $368 / 77$ & $0.902^{b}$ & $194 / 62$ & $0.227^{b}$ \\
\hline TNM (I/II/III or IV) & $38 / 42 / 4$ & $36 / 69 / 13$ & $0.054^{b}$ & $193 / 215 / 37$ & $0.536^{\mathrm{b}}$ & $87 / 130 / 39$ & $0.022^{\mathrm{b}}$ \\
\hline $\mathrm{BCLC}(\mathrm{A} / \mathrm{B})$ & $53 / 31$ & $81 / 37$ & $0.002^{b}$ & $3 / 2 / 133$ & $0.136^{\mathrm{b}}$ & $|55 /| 0 \mid$ & $0.191^{b}$ \\
\hline
\end{tabular}

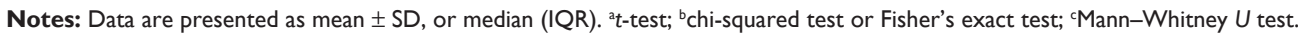

Abbreviations: AFP, $\alpha$-fetoprotein; ALB, albumin; ALP, alkaline phosphatase; ALT, alanine transaminase; AST, aspartate aminotransferase; BCLC, Barcelona Clinic for Liver Cancer; $\gamma$-GT, $\gamma$-glutamyltransferase; HBV, hepatitis B virus; HR, hepatic resection; MELD, Model for End-Stage Liver Disease Score; NLR, neutrophil-lymphocyte ratio; PT, prothrombin time; RFA, radiofrequency ablation; TACE, transcatheter arterial chemoembolization; TACE-RFA, TACE combined with RFA; TBIL, total bilirubin; TNM, tumor node metastasis staging. 
Table 2 Predictors of MVI in patients with HCC based on univariate and multivariate Cox regression analyses $(n=903)$

\begin{tabular}{|c|c|c|c|c|c|c|}
\hline \multirow[t]{2}{*}{ Variables } & \multicolumn{3}{|c|}{ Univariate Cox } & \multicolumn{3}{|c|}{ Multivariate Cox } \\
\hline & HR & $95 \% \mathrm{Cl}$ & $P$-value & HR & $95 \% \mathrm{Cl}$ & $P$-value \\
\hline Age & 0.980 & $(0.957-1.003)$ & 0.910 & & & \\
\hline Sex & 0.604 & $(0.309-1.180)$ & 0.140 & & & \\
\hline HBV related & 1.924 & $(0.833-4.444)$ & 0.126 & & & \\
\hline$\gamma$-GT & 1.002 & $(1.001-1.004)$ & 0.001 & 1.002 & $(1.000-1.003)$ & 0.025 \\
\hline TBIL & 1.004 & $(1.00 \mathrm{I}-1.006)$ & 0.008 & & & \\
\hline ALB & 0.945 & $(0.915-0.977)$ & 0.001 & 0.947 & $(0.9|4-0.98|)$ & 0.002 \\
\hline PT & 1.135 & $(1.039-1.240)$ & 0.005 & & & \\
\hline AFP $\geq 400 \mathrm{ng} / \mathrm{mL}$ & 2.215 & $(1.302-3.767)$ & 0.003 & 1.875 & $(1.079-3.257)$ & 0.026 \\
\hline Tumor number $\geq 3$ & 1.709 & $(1.04 I-2.804)$ & 0.034 & & & \\
\hline Tumor diameter $\geq 5 \mathrm{~cm}$ & 1.825 & $(1.080-3.086)$ & 0.025 & & & \\
\hline Intervention & $0.64 I$ & $(0.485-0.845)$ & 0.002 & $0.74 I$ & $(0.556-0.987)$ & 0.041 \\
\hline
\end{tabular}

Abbreviations: AFP, $\alpha$-fetoprotein; ALB, albumin; $\gamma$-GT, $\gamma$-glutamyltransferase; HBV, hepatitis B virus; HCC, hepatocellular carcinoma; MVI, macrovascular invasion; PT, prothrombin time; TBIL, total bilirubin.

revealed that $\gamma$-GT, ALB, TBIL, prothrombin time, AFP, tumor number, tumor diameter, and intervention were predictive factors for MVI. These factors were included in the multivariate Cox regression analyses. Furthermore, intervention, $\gamma$-GT, ALB, and AFP $\geq 400 \mathrm{ng} / \mathrm{mL}$ were selected as significant prognostic factors for MVI by forward selection (Table 2).

The predictive value of intervention, $\gamma$-GT, ALB, and $\mathrm{AFP} \geq 400 \mathrm{ng} / \mathrm{mL}$ for MVI prognosis was evaluated by comparing the ROC curves from the related parameters. Figure 1

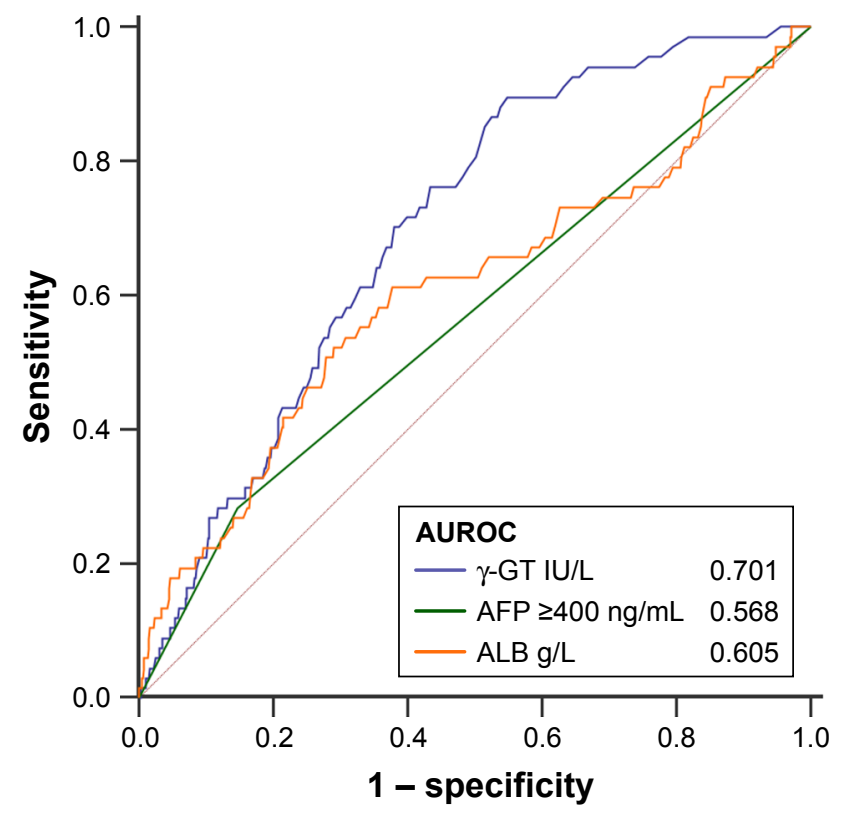

Figure I ROC curves for variables shown to be associated with MVI in multivariate analysis.

Note: The area under the curve of $\gamma$-GT developed in the study was greater than that of other predictive indicators.

Abbreviations: AFP, $\alpha$-fetoprotein; ALB, albumin; AUROC, area under ROC; $\gamma$-GT, $\gamma$-glutamyltransferase; MVI, macrovascular invasion; ROC, receiver operating characteristics. shows that the area under the ROC curve for $\gamma$-GT $(0.701)$ was higher than that for AFP (0.568) and ALB (0.605). These results indicate that the baseline $\gamma$-GT level in patients with $\mathrm{HCC}$ is an important predictive factor for MVI.

\section{Serum $\gamma$-GT level after different treatments}

The median (IQR) serum $\gamma$-GT before HR, TACE-RFA, or TACE was 36.6 (22.3-79.2), 46.6 (26.7-89.0), or 45.2 (26.4-86.1), respectively; the $\gamma$-GT level was 50.0 (29.3$88.2), 44.4$ (28.1-75.4), or $43.1(26.4-84.5)$ at 3 months after the corresponding interventions. The $\gamma$-GT level after HR was significantly higher than that before $\operatorname{HR}(P=0.037)$. No significant difference was found before and after TACERFA $(P=0.516)$ or TACE $(P=0.817)$.

\section{Determining the cutoff value for elevated $\gamma$-GT using the ROC curve}

Based on the ROC curve analysis, the optimal cutoff value of serum $\gamma$-GT was $39 \mathrm{IU} / \mathrm{L}$ for predicting the occurrence of MVI. The area under the ROC curve was 0.701 with a 95\% CI of $0.669-0.731$ (Figure 1). Sensitivity was $87.0 \%$ and specificity was $45.6 \%$ with some relation to the highest Youden index (sensitivity + specificity -1 ). Consequently, all intervention groups were divided according to $\gamma$-GT level: $\geq 39$ or $<39$ IU/L.

\section{MVI occurrence based on $\gamma$-GT levels after different interventions}

We analyzed the effect of baseline serum $\gamma$-GT levels on the occurrence of MVI. The rates of 1-year MVI after HR, TACE-RFA, or TACE were $8.9 \%, 8.6 \%$, or $17.1 \%$, respectively, in the $\gamma-\mathrm{GT} \geq 39 \mathrm{IU} / \mathrm{L}$ group, and $0.0 \%, 2.1 \%$, or $1.8 \%$, 

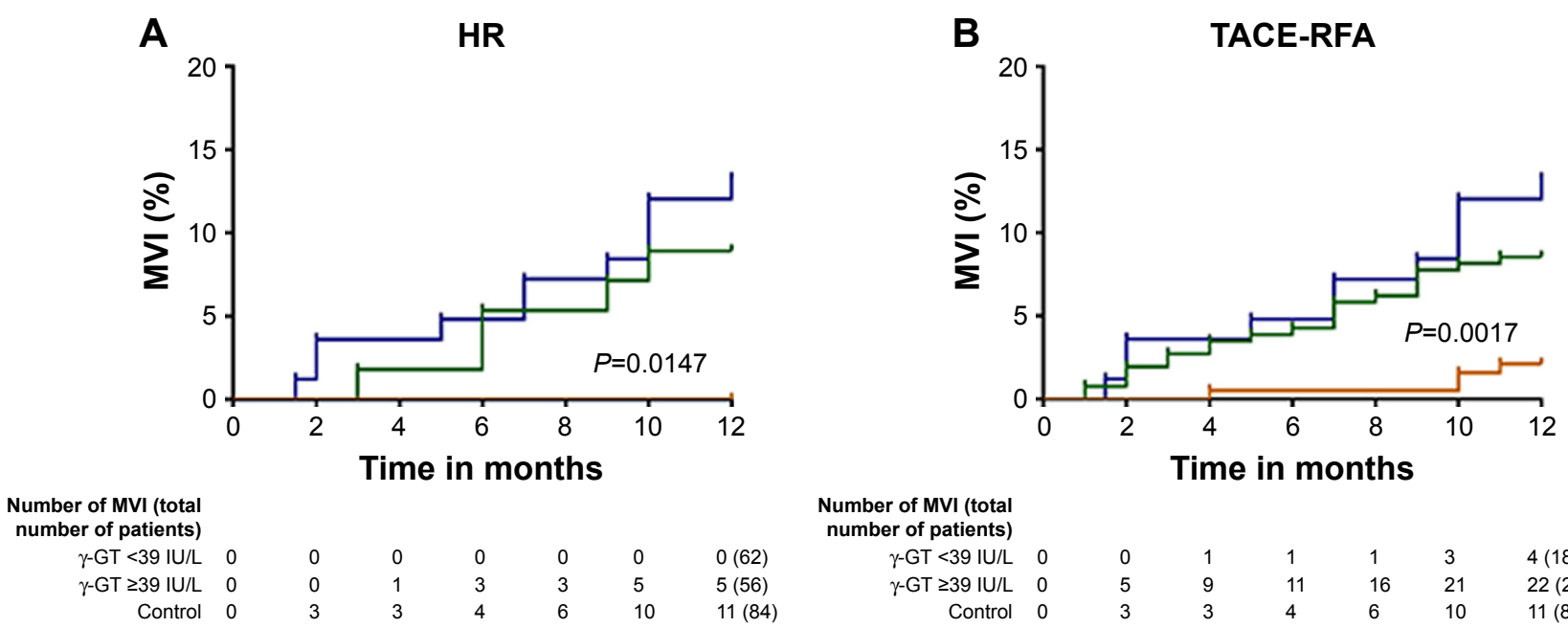

Number of MVI (total number of patients)

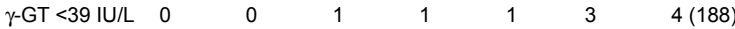

TACE

C

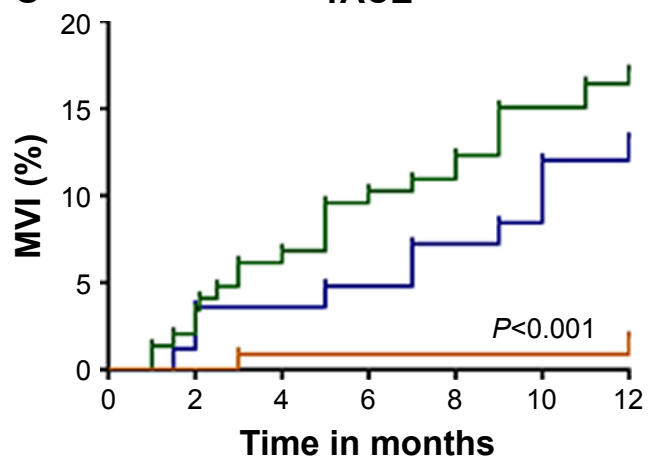

Number of MVI (total number of patients)

$\begin{array}{llllllll}\gamma-\mathrm{GT}<39 \mathrm{IU} / \mathrm{L} & 0 & 0 & 1 & 1 & 1 & 1 & 2(110)\end{array}$

$\begin{array}{llllllll}\gamma-\mathrm{GT} \geq 39 \mathrm{IU} / \mathrm{L} & 0 & 5 & 10 & 15 & 18 & 22 & 25(146)\end{array}$

$\begin{array}{llllllll}\text { Control } & 0 & 3 & 3 & 4 & 6 & 10 & 11(84)\end{array}$

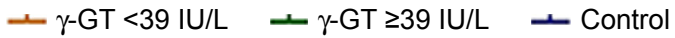

Figure 2 Kaplan-Meier analysis for the incidence of MVI after different interventions.

Notes: Kaplan-Meier survival curves demonstrating that patients with $\gamma$-GT $<39$ IU/L exhibited lower I-year MVI rates than those with $\gamma$-GT $\geq 39$ IU/L after (A) HR, (B) TACE-RFA, or (C) TACE ( $P=0.0147, P=0.0017$, and $P<0.001$, respectively).

Abbreviations: $\gamma$-GT, $\gamma$-glutamyltransferase; HR, hepatic resection; MVI, macrovascular invasion; TACE, transcatheter arterial chemoembolization; TACE-RFA, TACE combined with radiofrequency ablation.

respectively, in the $\gamma$-GT $<39 \mathrm{IU} / \mathrm{L}$ group $(P=0.0166$, $P=0.0041$, or $P<0.001$, respectively; Figure 2A-C). Correspondingly, there were no significant differences between the 1-year MVI rates after HR, TACE-RFA, or TACE in the $\gamma-\mathrm{GT} \geq 39 \mathrm{IU} / \mathrm{L}$ group and the control group (13.1\%) $(P=0.4402, P=0.2214$, or $P=0.4159$, respectively; Figure $2 \mathrm{~A}-\mathrm{C})$. The results showed that a baseline serum $\gamma$-GT $\geq 39$ IU/L was related to obviously higher MVI rates than $\gamma$-GT $<39$ IU/L at 1 year in patients with HCC who underwent HR, TACE-RFA, or TACE. MVI rates were similar at 1 year in the $\gamma-\mathrm{GT} \geq 39 \mathrm{IU} / \mathrm{L}$ group that underwent HR, TACE-RFA, or TACE and in the control group.

Compared to the effects of different treatments in $\gamma$-GT $<39$ IU/L and $\gamma$-GT $\geq 39$ IU/L groups on the occurrence of MVI, the results showed that the difference in the effects of various treatments in $\gamma$-GT $<39$ IU/L group on the occurrence of MVI was not significant $(P=0.5167)$ (Figure 3A). However, the incidence of MVI after TACE was significantly higher than that after HR or TACE-RFA in $\gamma$-GT $\geq 39 \mathrm{IU} / \mathrm{L}$ group $(P=0.0253$; Figure $3 \mathrm{~B})$.

\section{Subgroup of MVI occurrence based on BCLC stage}

We further clarified the prognostic value of baseline serum $\gamma$-GT for 1-year MVI occurrence in patients with BCLC A or B stage HCC. Our results demonstrated that in BCLC A stage, after HR, TACE-RFA, or TACE the 1-year MVI rates were $0.0 \%, 0.7 \%$, or $2.6 \%$ for patients with $\gamma$-GT $<39 \mathrm{IU} / \mathrm{L}$ and $14.7 \%, 6.7 \%$, or $10.4 \%$ for patients with $\gamma$-GT $\geq 39 \mathrm{IU} / \mathrm{L}(P=0.0067, P=0.0060$, or $P=0.0464$, 


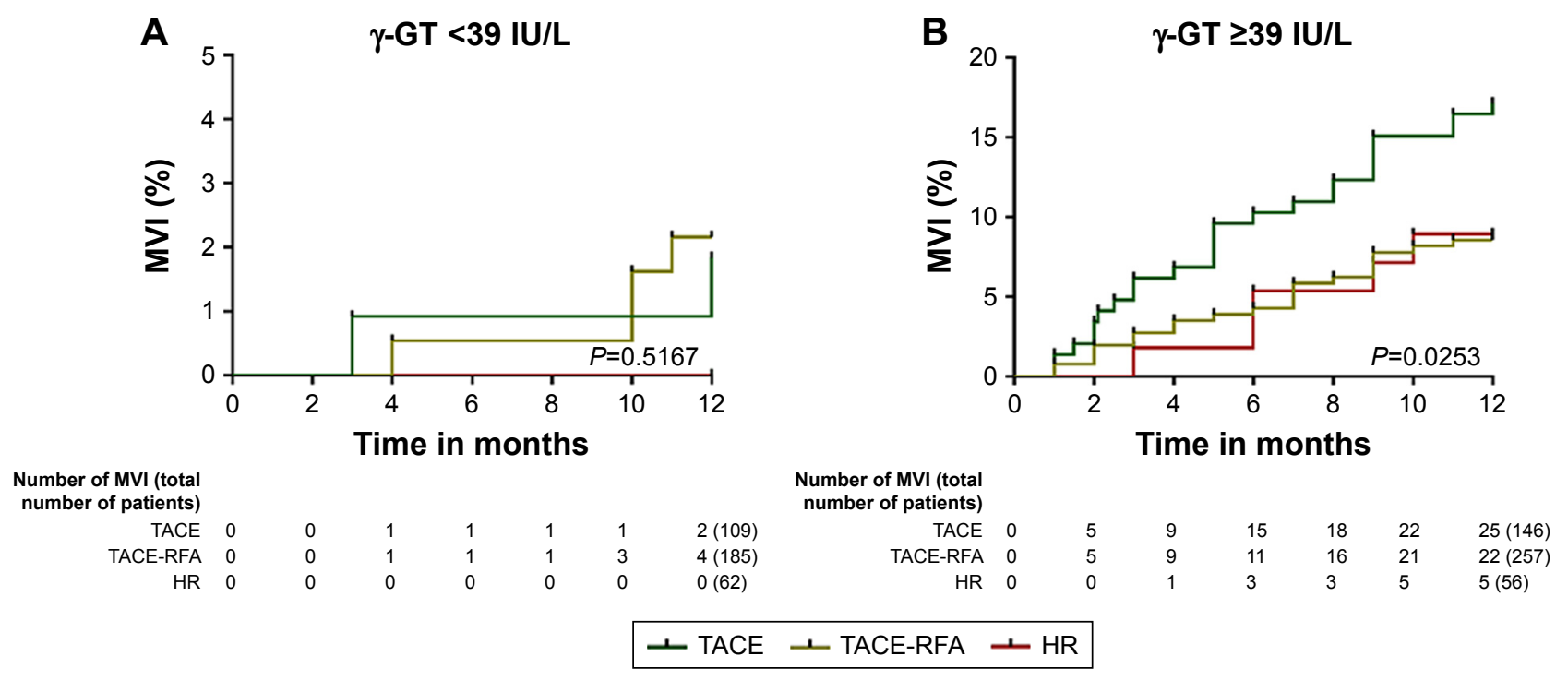

Figure 3 The MVI incidences after the $\gamma$-GT stratification.

Notes: Kaplan-Meier survival curves demonstrating that the difference in the effects of various treatments in $\gamma$-GT $<39$ IU/L group (A) on the occurrence of MVI was not significant $(P=0.5167)$. In $\gamma-G T \geq 39 \mathrm{IU} / \mathrm{L}$ group $(B)$, the incidence of $\mathrm{MVI}$ after TACE was significantly higher than those after HR or TACE-RFA ( $P=0.0253)$.

Abbreviations: $\gamma$-GT, $\gamma$-glutamyltransferase; HR, hepatic resection; MVI, macrovascular invasion; TACE, transcatheter arterial chemoembolization; TACE-RFA, TACE combined with radiofrequency ablation.

respectively; Figure $4 \mathrm{~A}, \mathrm{~B}$, and $\mathrm{D})$. In the BCLC B stage, after TACE-RFA, the MVI rates were $7.1 \%$ for patients with $\gamma-\mathrm{GT}<39 \mathrm{IU} / \mathrm{L}$ and $11.7 \%$ for patients with $\gamma$-GT $\geq 39 \mathrm{IU} / \mathrm{L}$. The Kaplan-Meier analysis showed that the MVI rates were similar at 1 year $(P=0.4033$; Figure $4 C)$. In the BCLC B stage, after TACE, the MVI rate was significantly lower in the $\gamma$-GT $<39$ IU/L group $(0.0 \%)$ than in the $\gamma$-GT $\geq 39 \mathrm{IU} / \mathrm{L}$ group $(25.4 \%)$ at 1 year $(P=0.0017$; Figure $4 \mathrm{E})$.

\section{Discussion}

Resection and liver transplantation are potentially curative treatments for $\mathrm{HCC}$, although most patients with $\mathrm{HCC}$ are diagnosed too late for curative treatments and receive only palliative treatment owing to an advanced tumor or severe underlying liver cirrhosis. ${ }^{31,32}$ In addition, there are also obvious differences in prognosis even for patients in the same phase owing to tumor and patient heterogeneity. Therefore, based on the BCLC phase, it is crucial to choose and monitor appropriate serum biochemical markers that can represent the tumor burden and underlying hepatic function.

Our multivariate analysis results showed that $\gamma$-GT was an independent predictive factor for MVI in patients with HCC without MVI. When we divided the patients into groups based on different treatments, we found that $\gamma$-GT offered good predictions for the MVI in the HR, TACE-RFA, and TACE groups. An abnormal baseline serum $\gamma$-GT level was related to significantly higher rates of MVI at 1 year, similar to the control group. The effects of different treatments in $\gamma$-GT $<39$ IU/L and $\gamma$-GT $\geq 39$ IU/L groups on the occurrence of MVI were further compared. The incidence of MVI after TACE was significantly higher than those after HR or TACE-RFA in $\gamma$-GT $\geq 39$ IU/L group. Furthermore, in subgroups of BCLC stages, $\gamma$-GT could still differentiate patients with a lower MVI occurrence from patients with a higher MVI occurrence in patients with HCC in the BCLC A stage. Moreover, in patients with $\mathrm{HCC}$ in the BCLC B stage who received TACE, $\gamma$-GT $<39$ IU/L was associated with lower MVI occurrence.

The $\gamma$-GT level, in particular, has critical clinical significance as an indicator of prognosis. ${ }^{33,34}$ This indicator helps to predict prognosis and helps with the selection of further treatment. Several studies revealed that the preoperative $\gamma$-GT level is a reliable prognostic biomarker with a bright future in patients with HCC who underwent HR. ${ }^{25,35,36}$ The $\gamma$-GT level also was a crucial prognostic factor predicting prognosis of patients with HCC treated with TACE, RFA, or liver transplantation. ${ }^{26-28,37}$ However, in most of the above studies, the outcome of interest was overall survival, recurrence, and/or disease-free survival, and there had been no research on the outcome of MVI occurrence. The reason our study established 1-year MVI occurrence as an outcome was because MVI has a significant adverse influence on prognosis, with an observably decreased median survival time. ${ }^{6-8}$

Our study has the following limitations. First, the presence or absence of microscopic vascular invasion diagnosed by pathological examination at baseline was not discriminated, which may influence the occurrence of MVI. However, this was a retrospective study and pathological data 


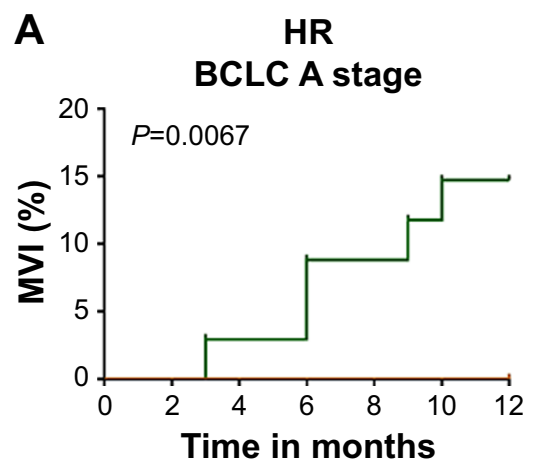

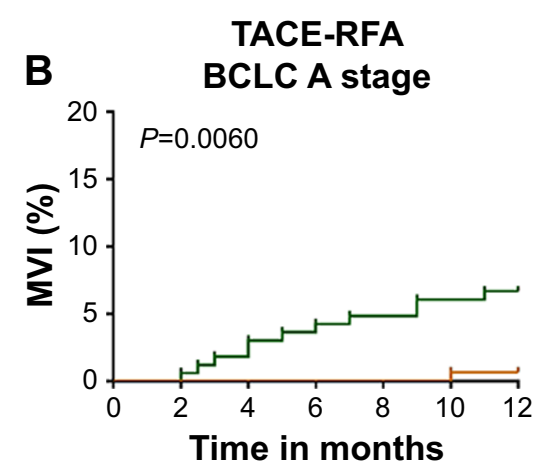

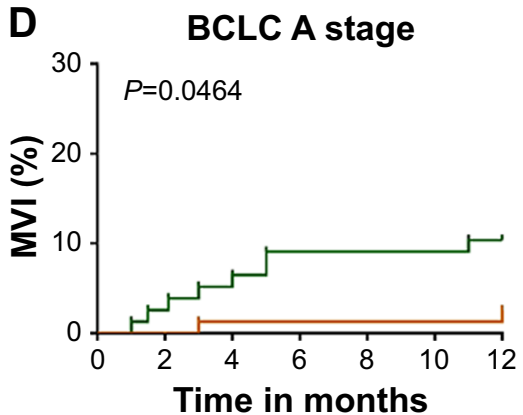

TACE-RFA
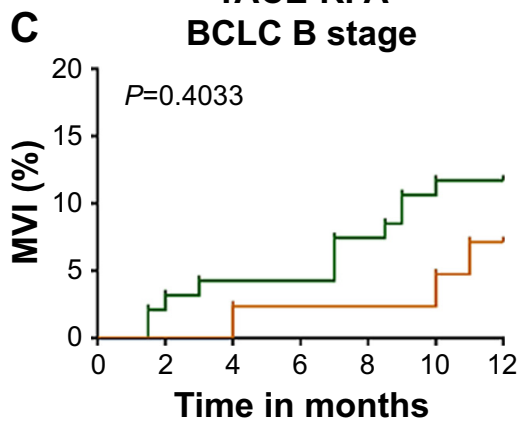

E

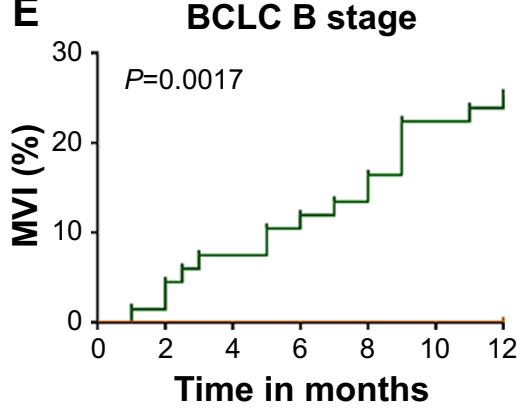

$-\gamma-\mathrm{GT}<39 \mathrm{IU} / \mathrm{L} \quad \gamma-\gamma \mathrm{GT} \geq 39 \mathrm{IU} / \mathrm{L}$

Figure 4 The $M V I$ incidences of BCLC A and B stage.

Notes: Kaplan-Meier survival curves demonstrating that patients with $\gamma$-GT $<39$ IU/L exhibited lower I-year MVI rates than those with $\gamma$-GT $\geq 39$ IU/L in the BCLC A stage after (A) HR, (B) TACE-RFA, or (D) TACE, and (E) in the BCLC B stage after TACE ( $P=0.0067, P=0.0060, P=0.0464$, and $P=0.0017$, respectively); the MVI rates were similar at I year for patients with $\gamma-\mathrm{GT}<39 \mathrm{IU} / \mathrm{L}$ and $\gamma-\mathrm{GT} \geq 39 \mathrm{IU} / \mathrm{L}$ in the BCLC B stage after (C) TACE-RFA (P=0.4033).

Abbreviations: BCLC, Barcelona Clinic Liver Cancer; $\gamma$-GT, $\gamma$-glutamyltransferase; HR, hepatic resection; MVI, macrovascular invasion; TACE, transcatheter arterial chemoembolization; TACE-RFA, TACE combined with radiofrequency ablation.

could not be collected. It was inevitable of other selections, withdrawals, and other clinical bias because our study was retrospective. Second, the source of all collected data was a single medical center with a limited sample size. Therefore, in the future, prospective clinical studies with larger sample sizes are needed to confirm our study results and promote the clinical application of $\gamma$-GT.

\section{Conclusion}

Baseline serum $\gamma$-GT stratification may help select the appropriate treatment to reduce the incidence of MVI, which may be expected to lead to a novel strategy that decreases the incidence of MVI and indirectly prolongs survival after different interventions in patients with HCC.

\section{Author contributions}

All authors contributed to data analysis, drafting and revising the article, gave final approval of the version to be published, and agree to be accountable for all aspects of the work.

\section{Disclosure}

The author reports no conflicts of interest in this work. 


\section{References}

1. Torre LA, Bray F, Siegel RL, Ferlay J, Lortet-Tieulent J, Jemal A. Global Cancer statistics, 2012. CA Cancer J Clin. 2015;65(2):87-108.

2. Reddy SK, Barbas AS, Turley RS, et al. Major liver resection in elderly patients: a multi-institutional analysis. J Am Coll Surg. 2011; 212(5):787-795.

3. Rich N, Singal AG. Hepatocellular carcinoma tumour markers: current role and expectations. Best Pract Res Clin Gastroenterol. 2014;28(5): 843-853.

4. Forner A, Llovet JM, Bruix J. Hepatocellular carcinoma. Lancet. 2012;379(9822):1245-1255.

5. Jonas S, Bechstein WO, Steinmüller T, et al. Vascular invasion and histopathologic grading determine outcome after liver transplantation for hepatocellular carcinoma in cirrhosis. Hepatology. 2001; 33(5):1080-1086.

6. Minagawa M, Makuuchi M. Treatment of hepatocellular carcinoma accompanied by portal vein tumor thrombus. World J Gastroenterol. 2006;12(47):7561-7567.

7. Llovet JM, Bustamante J, Castells A, et al. Natural history of untreated nonsurgical hepatocellular carcinoma: rationale for the design and evaluation of therapeutic trials. Hepatology. 1999;29(1):62-67.

8. Schöniger-Hekele M, Müller C, Kutilek M, Oesterreicher C, Ferenci P, Gangl A. Hepatocellular carcinoma in central Europe: prognostic features and survival. Gut. 2001;48(1):103-109.

9. $\mathrm{Xu} \mathrm{X}, \mathrm{Lu} \mathrm{D}$, Ling Q, et al. Liver transplantation for hepatocellular carcinoma beyond the Milan criteria. Gut. 2016;65(6):1035-1041.

10. Zhu WJ, Huang CY, Li C, et al. Risk factors for early recurrence of HBV-related hepatocellular carcinoma meeting Milan criteria after curative resection. Asian Pac J Cancer Prev. 2013;14(12):7101-7106.

11. Yang SL, Liu LP, Yang S, et al. Preoperative serum $\alpha$-fetoprotein and prognosis after hepatectomy for hepatocellular carcinoma. Br J Surg. 2016; 103(6):716-724.

12. Fu SJ, Qi CY, Xiao WK, Li SQ, Peng BG, Liang LJ. Glypican-3 is a potential prognostic biomarker for hepatocellular carcinoma after curative resection. Surgery. 2013;154(3):536-544.

13. Zhou L, Rui JA, Wang SB, Chen SG, Qu Q. Risk factors of microvascular invasion, portal vein tumor thrombosis and poor post-resectional survival in HBV-related hepatocellular carcinoma. Hepatogastroenterology. 2014;61(134):1696-1703.

14. Chen C, Chen DP, Gu YY, et al. Vascular invasion in hepatitis B virusrelated hepatocellular carcinoma with underlying cirrhosis: possible associations with ascites and hepatitis B viral factors? Tumour Biol. 2015;36(8):6255-6263.

15. Zheng J, Seier K, Gonen M, et al. Utility of serum inflammatory markers for predicting microvascular invasion and survival for patients with hepatocellular carcinoma. Ann Surg Oncol. 2017;24(12):3706-3714.

16. You Z, Chen LP, Ye H. Predictors of microvascular invasion in patients with solitary small hepatitis B related hepatocellular carcinoma. Pak J Med Sci. 2014;30(2):331-334.

17. Orlowski M, Meister A. The gamma-glutamyl cycle: a possible transport system for amino acids. Proc Natl Acad Sci U S A. 1970;67(3): 1248-1255.

18. Teschke R, Rauen J, Neuefeind M, Petrides AS, Strohmeyer G. Alcoholic liver disease associated with increased gamma-glutamyltransferase activities in serum and liver. Adv Exp Med Biol. 1980;132:647-654.

19. Lee DH, Silventoinen K, Jacobs DR, Jousilahti P, Tuomileto J. Gamma-glutamyltransferase, obesity, and the risk of type 2 diabetes: observational cohort study among 20,158 middle-aged men and women. J Clin Endocrinol Metab. 2004;89(11):5410-5414.
20. Ishizaka N, Ishizaka Y, Toda E, Yamakado M, Koike K, Nagai R. Association between gamma-glutamyltransferase levels and insulin resistance according to alcohol consumption and number of cigarettes smoked. J Atheroscler Thromb. 2010;17(5):476-485.

21. Dominici S, Pieri L, Comporti M, Pompella A. Possible role of membrane gamma-glutamyltransferase activity in the facilitation of transferrin-dependent and -independent iron uptake by cancer cells. Cancer Cell Int. 2003;3(1):7.

22. Corti A, Franzini M, Paolicchi A, Pompella A. Gamma-glutamyltransferase of cancer cells at the crossroads of tumor progression, drug resistance and drug targeting. Anticancer Res. 2010;30(4):1169-1181.

23. Whitfield JB. Gamma glutamyl transferase. Crit Rev Clin Lab Sci. 2001; 38(4):263-355.

24. Pompella A, De Tata V, Paolicchi A, Zunino F. Expression of gammaglutamyltransferase in cancer cells and its significance in drug resistance. Biochem Pharmacol. 2006;71(3):231-238.

25. Fu S, Guo Z, Li S, et al. Prognostic value of preoperative serum gammaglutamyltranspeptidase in patients with hepatocellular carcinoma after hepatectomy. Tumour Biol. 2016;37(3):3433-3440.

26. Zhang JB, Chen Y, Zhang B, et al. Prognostic significance of serum gamma-glutamyl transferase in patients with intermediate hepatocellular carcinoma treated with transcatheter arterial chemoembolization. Eur J Gastroenterol Hepatol. 2011;23(9):787-793.

27. Guiu B, Deschamps F, Boulin M, et al. Serum gamma-glutamyltransferase independently predicts outcome after transarterial chemoembolization of hepatocellular carcinoma: external validation. Cardiovasc Intervent Radiol. 2012;35(5):1102-1108.

28. $\mathrm{Ma} \mathrm{H}$, Zhang L, Tang B, et al. $\gamma$-Glutamyltranspeptidase is a prognostic marker of survival and recurrence in radiofrequency-ablation treatment of hepatocellular carcinoma. Ann Surg Oncol. 2014;21(9):3084-3089.

29. Bruix J, Sherman M; American Association for the Study of Liver Diseases. Management of hepatocellular carcinoma: an update. Hepatology. 2011;53(3):1020-1022.

30. Shah ZK, McKernan MG, Hahn PF, Sahani DV. Enhancing and expansile portal vein thrombosis: value in the diagnosis of hepatocellular carcinoma in patients with multiple hepatic lesions. AJR Am J Roentgenol. 2007;188(5):1320-1323.

31. Lencioni R. Loco-regional treatment of hepatocellular carcinoma. Hepatology. 2010;52(2):762-773.

32. Kim WR, Gores GJ, Benson JT, Therneau TM, Melton LJ. Mortality and hospital utilization for hepatocellular carcinoma in the United States. Gastroenterology. 2005;129(2):486-493.

33. Carr BI, Guerra V, Giannini EG, et al. Low alpha-fetoprotein HCC and the role of GGTP. Int J Biol Markers. 2014;29(4):395-402.

34. Sun L, Wang R, Gao FY, et al. Predictive value of serum gammaglutamyltransferase levels in patients with hepatocellular carcinoma. Neoplasma. 2017;64(3):444-452.

35. Wu SJ, Lin YX, Ye H, Xiong XZ, Li FY, Cheng NS. Prognostic value of alkaline phosphatase, gamma-glutamyl transpeptidase and lactate dehydrogenase in hepatocellular carcinoma patients treated with liver resection. Int J Surg. 2016;36(Pt A):143-151.

36. Song P, Inagaki Y, Wang Z, et al. High Levels of Gamma-Glutamyl Transferase and Indocyanine Green Retention Rate at 15 min as Preoperative Predictors of Tumor Recurrence in Patients With Hepatocellular Carcinoma. Medicine. 2015;94(21):e810.

37. Fu SJ, Zhao Q, Ji F, et al. Elevated preoperative serum gammaglutamyltranspeptidase predicts poor prognosis for hepatocellular carcinoma after liver transplantation. Sci Rep. 2016;6:28835. 


\section{Publish your work in this journal}

OncoTargets and Therapy is an international, peer-reviewed, open access journal focusing on the pathological basis of all cancers, potential targets for therapy and treatment protocols employed to improve the management of cancer patients. The journal also focuses on the impact of management programs and new therapeutic agents and protocols on
Dovepress

patient perspectives such as quality of life, adherence and satisfaction. The manuscript management system is completely online and includes a very quick and fair peer-review system, which is all easy to use. Visit http://www.dovepress.com/testimonials.php to read real quotes from published authors.

Submit your manuscript here: http://www.dovepress.com/oncotargets-and-therapy-journal 